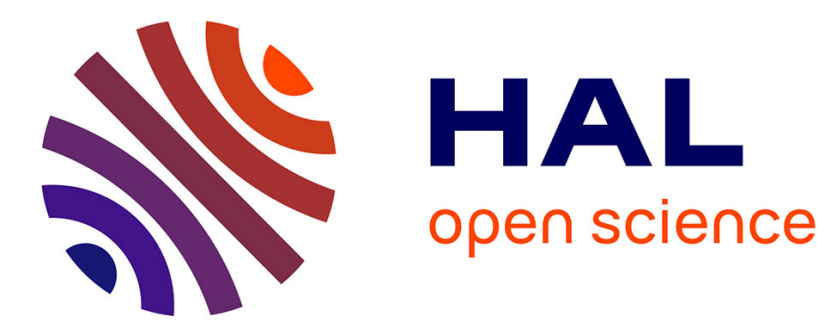

\title{
Absorption, émission et réflexion du rayonnement électromagnétique par une paroi constituée de tubes parallèles jointifs
}

\author{
A. Soufiani
}

\section{- To cite this version:}

A. Soufiani. Absorption, émission et réflexion du rayonnement électromagnétique par une paroi constituée de tubes parallèles jointifs. Revue de Physique Appliquée, 1983, 18 (1), pp.19-27. 10.1051/rphysap:0198300180101900 . jpa-00245063

\section{HAL Id: jpa-00245063 https://hal.science/jpa-00245063}

Submitted on 1 Jan 1983

HAL is a multi-disciplinary open access archive for the deposit and dissemination of scientific research documents, whether they are published or not. The documents may come from teaching and research institutions in France or abroad, or from public or private research centers.
L'archive ouverte pluridisciplinaire $\mathbf{H A L}$, est destinée au dépôt et à la diffusion de documents scientifiques de niveau recherche, publiés ou non, émanant des établissements d'enseignement et de recherche français ou étrangers, des laboratoires publics ou privés. 


\title{
Absorption, émission et réflexion du rayonnement électromagnétique par une paroi constituée de tubes parallèles jointifs
}

\author{
A. Soufiani \\ C.N.R.S. Equipe GESER (*), Ecole Centrale des Arts et Manufactures, Grande Voie des Vignes, \\ 92290 Chatenay-Malabry, France
}

(Reçu le ler avril 1982, révisé le 6 octobre 1982, accepté le 14 octobre 1982)

Résumé. - On se propose dans cette étude d'évaluer l'influence des deux phénomènes suivants :

1) macrorugosité d'une surface constituée de demi-tubes jointifs ;

2) gradients locaux de température engendrés par un flux incident normal au plan des génératrices, sur la nature de l'émission et de l'absorption du rayonnement par ce faisceau de tubes. Cette étude permet de définir une température équivalente radiative que l'on exprime simplement en fonction des paramètres du problème afin d'en avoir une utilisation facile par la suite.

\begin{abstract}
This paper describes an investigation of the effects of :
1) macro-rugosity of a wall constituted with joined parallel tubes;

2) local drops in temperature due to an incident flux normal to the generating lines, on the nature of emission and absorption of radiation by this bundle of tubes. As a result, an equivalent radiative temperature is defined. This temperature is simply expressed as a function of the various parameters of the system in order to be used easily afterwards.
\end{abstract}

\section{Nomenclatures}

$A_{p}, B_{p}$, : coefficients de la décomposition en série $A_{p}^{\prime}, B_{p}^{\prime} \quad$ de Fourier.

$a_{n}, b_{n} \quad$ : coefficients de la décomposition de $\phi(\theta)$ en série de Fourier.

$B=\frac{h R_{\mathrm{i}}}{k}$ : nombre sans dimension.

$B_{\mathrm{r}} \quad$ : proportionnel à une résistance thermique.

$e \quad:$ épaisseur du tube.

$E_{\mathrm{v}}(\theta) \quad$ : éclairement visible en 1 point.

$E_{v}^{*}(\theta) \quad$ : éclairement visible adimensionnel.

$h \quad$ : coefficient d'échange convectif.

$J(\theta) \quad$ : radiosité infrarouge en 1 point.

$k \quad:$ : conductivité thermique du tube.

$L(\theta) \quad$ : luminance du flux $J(\theta)$.

$r \quad$ : rayon dans le tube.

$R_{\mathrm{i}} \quad$ : rayon interne.

$R_{\mathrm{e}} \quad$ : rayon externe.

$T_{\mathrm{v}} \quad$ : température de veine fluide.

$T(r, \theta) \quad$ : température en 1 point du tube.

$T_{\mathrm{i}}(\theta) \quad$ : température en $r=R_{\mathrm{i}}$.

$T_{\mathrm{e}}(\theta) \quad$ : température en $r=R_{\mathrm{e}}$.
$T_{\text {rad }}$ : température équivalente radiative.

$T_{\mathrm{cc}} \quad$ : température conductive convective.

\section{LETTRES GRECQUES.}

$\alpha \quad$ : proportionnelle à une résistance thermique.

$\alpha_{\mathrm{v}} \quad$ : absorptivité dans le visible.

$\alpha_{\text {ir }} \quad$ : absorptivité dans l'infrarouge.

$\beta \quad$ : proportionnelle à une température.

$\gamma \quad$ : angle caractérisant la direction de l'émission.

$\delta \quad: \quad$ angle entre la normale locale et la direction de l'émission.

$\delta F_{\mathrm{d} S_{1} \rightarrow \mathrm{d} S_{2}}:$ facteur de forme géométrique.

$\varepsilon_{\text {ir }} \quad:$ émissivité dans l'infrarouge.

$\mu \quad$ : nombre sans dimension $=r / R_{\mathrm{i} \text {. }}$

$\mu_{n} \quad: R_{\mathrm{e}} / \boldsymbol{R}_{\mathrm{i}}$.

$\phi_{0} \quad$ : flux incident.

$\phi_{v}(\theta) \quad$ : flux visible absorbé en 1 point.

$\phi_{\mathrm{ir}}(\theta) \quad$ : flux infrarouge absorbé en 1 point.

$\phi(\theta) \quad$ : flux global absorbé en 1 point.

$\mathrm{d} \phi / \mathrm{d} \gamma \quad$ : flux directionnel émis.

$\phi_{\mathrm{t}} \quad$ : flux total émis.

$\phi_{\text {abs }} \quad$ : flux global moyen absorbé.

$\psi(\mu, \theta) \quad$ : température adimensionnelle.

$\sigma \quad$ : constante de Stefan-Boltzmann.

$\theta \quad:$ angle par rapport à la normale moyenne.

(*) En relation avec le Groupe de Recherche Thermique. 
INDICES.

$\mathrm{v} \quad$ : visible.

ir $\quad:$ infrarouge.

1,2 : tube 1 ou 2.

$\left\{\begin{array}{l}\text { i } \\ \text { s } \\ \text { inf } \\ \text { sup }\end{array}\right.$

$\left\{\begin{array}{l}p \\ n\end{array} \quad\right.$ : indices de séries.

1. Introduction. - L'influence des macrorugosités d'une surface sur ses caractéristiques radiatives (facteur d'émission ou d'absorption) a déjà été étudiée pour plusieurs géométries que l'on rencontre couramment (voir par exemple Réf. [1]). Mais à notre connaissance, la surface qui absorbe ou qui émet a toujours été supposée isotherme. Or, un flux quelconque concentré sur une surface tubulaire par exemple, peut créer d'importants gradients de température dans un tube, surtout quand sa conductivité thermique est faible. Ce phénomène intervient couramment dans les chaudières classiques et dans les récepteurs solaires à parois tubulaires où l'énergie électromagnétique, après concentration dans un système optique quelconque (champ d'héliostats, paraboloïdes, ...) arrive sur le tube avec une répartition proportionnelle au cosinus de l'angle que fait le rayon incident avec la normale locale, ce qui crée des différences de température avec un maximum situé au sommet du tube, lequel va donc émettre plus que les autres parties.

Le but de l'étude comporte deux points :

a) L'évaluation exacte de la répartition de la puissance absorbée par le tube dans le visible et dans l'infrarouge le long du périmètre de sa section droite, ainsi que le calcul des puissances directionnelles et de la puissance totale émise par la surface, compte tenu de sa macrorugosité et des gradients thermiques importants qu'elle peut engendrer.

b) La détermination d'une température équivalente radiative définie comme étant celle qu'aurait une surface plane, ayant le même facteur d'émission, pour émettre la même puissance que la surface tubulaire. Cette notion permet de remplacer celle d'émissivité apparente (que l'on ne peut plus définir à cause de l'anisothermie de la surface) afin de caractériser l'émission de la paroi. Elle est aussi destinée à l'interprétation des mesures des températures de paroi que l'on peut faire à distance par thermographie infrarouge.

En effet, étant données les fortes valeurs des flux solaires qu'on est amené à considérer, il est souvent difficile pour l'expérimentation, d'avoir une mesure directe de la répartition des températures de paroi; par contre, il est plus aisé de faire une mesure à distance du flux moyen émis par un paquet de tubes grâce à une caméra infrarouge, ce qui nous permet de remon- ter facilement à la température équivalente radiative définie précédemment.

2. Analyse du problème. - Considérons une surface constituée de demi-tubes jointifs et coaxiaux recevant un flux incident $\phi_{0}$ ne variant pas le long des génératrices (voir Fig. 1).

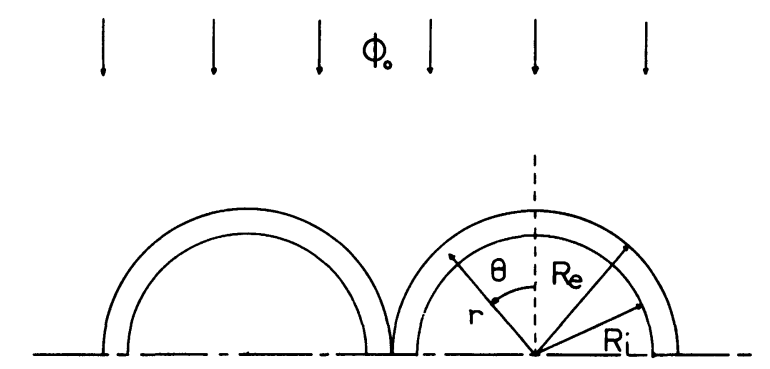

Fig. 1. - Géométrie tubulaire.

[Tubular geometry.]

Nous ferons les hypothèses suivantes :

- Les tubes seront supposés de longueur infinie de telle sorte que toutes les grandeurs que nous aurons à utiliser ne varient pas le long des génératrices.

- Les tubes ainsi que le fluide caloporteur sont opaques au rayonnement : l'échange thermique entre les deux est réalisé uniquement par convection forcée.

- Le coefficient de transfert convectif $h$ métalfluide est supposé constant tout le long de la paroi interne du tube, ainsi la chaleur évacuée est proportionnelle localement à $h\left(T_{\mathrm{i}}(\theta)-T_{\mathrm{v}}\right)$, où $T_{\mathrm{v}}$ est la température de veine fluide supposée connue et $T_{\mathrm{i}}(\theta)$ la température métal interne en un point repéré par l'angle $\theta$.

- Tous les tubes sont supposés parfaitement similaires (faisceau formé d'un grand nombre de tubes).

- Sur le plan radiatif, les caractéristiques de la surface externe sont supposées connues et indépendantes de la température.

- L'émission et la réflexion locales sont supposées isotropes.

- Enfin, nous supposerons que cette surface externe est semi-grise, c'est-à-dire que nous considérons deux bandes spectrales, l'une dite visible recouvrant l'intervalle $0,3 \mu \mathrm{m}-2,5 \mu \mathrm{m}$, et contenant tout le flux solaire, l'autre dite infrarouge allant de 2,5 à $20 \mu \mathrm{m}$, et contenant, pour le domaine de température considéré, tout le flux émis par les tubes de façon à utiliser des facteurs d'absorption et d'émission moyennés qu'on considérera constants à l'intérieur de chacune des deux bandes.

Compte tenu de toutes ces hypothèses, le calcul se déroulera en deux étapes :

1) d'abord le traitement du "visible " avec les réflexions successives qu'il peut y avoir entre deux 
tubes voisins, ce qui conduira à une répartition angulaire du flux visible absorbé.

2) traitement de l'infrarouge : le calcul du flux infrarouge absorbé en un point ou échangé entre deux tubes voisins nécessite au préalable la connaissance des températures tout le long de la paroi externe. Or ces températures, elles-mêmes, ne pourront être déterminées que si l'on connaît la condition limite du problème conductif et qui est justement la répartition angulaire du flux total absorbé. D'où la nécessité d'un calcul itératif comme nous le verrons plus loin.

2. 1 CALCUL DE L'ABSORPTION DANS LE « VISIBLE ». Soit $E_{\mathrm{v} 1}\left(\theta_{1}\right)$ l'éclairement visible au point $\mathbf{M}_{1}$ d'angle $\theta_{1}$, le flux visible absorbé en $\mathbf{M}_{1}$ s'écrit :

$$
\phi_{\mathrm{v}}\left(\theta_{1}\right)=\alpha_{\mathrm{v}} E_{\mathrm{v} 1}\left(\theta_{1}\right)
$$

où $\alpha_{\mathrm{v}}$ est le facteur d'absorption dans le visible.

$E_{\mathrm{v} 1}\left(\theta_{1}\right)$ est la somme du flux direct incident : $\phi_{0} \cos \left(\theta_{1}\right)$ et du flux qui après réflexion sur le tube 2 atteint $\mathbf{M}_{1}$, on peut donc écrire d'après l'hypothèse d'isotropie de la réflexion locale et d'après la relation de réciprocité des facteurs de forme :

$E_{\mathrm{v} 1}\left(\theta_{1}\right)=\phi_{0} \cos \theta_{1}+\left(1-\alpha_{\mathrm{v}}\right) \int_{\theta_{2 \mathrm{inf}}}^{\theta_{2} \text { up }} E_{\mathrm{v} 2}\left(\theta_{2}\right) \delta F_{\mathrm{d} S_{1} \rightarrow \mathrm{d} S_{2}}$

$\theta_{2 \text { inf }}$ et $\theta_{2 \text { sup }}$ sont les angles limites vus de $\mathbf{M}_{1}$ (Fig. 2) et ils sont donnés par les relations géométriques :

$\theta_{2 \text { inf }}=\frac{\pi}{2}-\operatorname{Arcsin}\left(\frac{\cos \theta_{1}+2\left(2-\sin \theta_{1}\right) \sqrt{1-\sin \theta_{1}}}{5-4 \sin \theta_{1}}\right)$

$\theta_{2 \text { sup }}=\frac{\pi}{2}-\theta_{1}+\operatorname{Arcsin}\left(2 \sin \theta_{1}-1\right)$

$\mathrm{d} S_{1}$ et $\mathrm{d} S_{2}$ sont des bandes élémentaires de longueur infinie contenant $\mathrm{M}_{1}$ et $\mathrm{M}_{2}, E_{\mathrm{v}_{2}}\left(\theta_{2}\right)$ est l'éclairement visible en $\mathrm{M}_{2}$, et enfin $\delta F_{\mathrm{d} S_{1} \rightarrow \mathrm{d} S_{2}}$ désigne le facteur de forme géométrique de $\mathrm{d} S_{1}$ vers $\mathrm{d} S_{2}$ qu'on peut calculer par les relations suivantes (Réf. [1]) :

$$
\delta F_{\mathrm{d} S_{1} \rightarrow \mathrm{d} S_{2}}=\frac{1}{2} \delta(\sin \varphi)=\frac{1}{2} \frac{|d . \delta a-a . \delta d|}{d^{2}}
$$

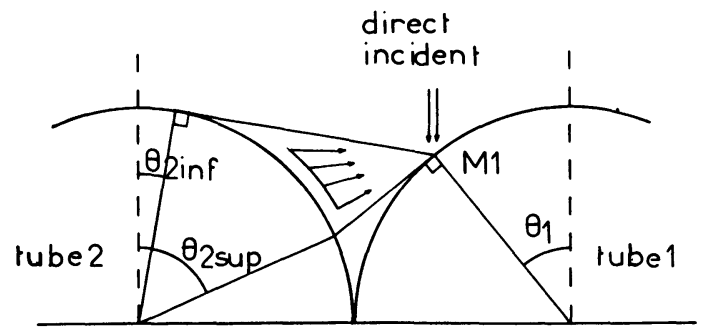

Fig. 2. - Angles limites.

[Limit angles.] avec (Fig. 3) :

$$
\begin{aligned}
d & =2 R_{\mathrm{e}}\left(\frac{3}{2}-\frac{1}{2} \cos \left(\theta_{1}+\theta_{2}\right)-\sin \theta_{1}-\sin \theta_{2}\right)^{1 / 2} \\
\delta d & =\frac{R_{\mathrm{e}}^{2}}{d}\left(\sin \left(\theta_{1}+\theta_{2}\right)-2 \cos \theta_{2}\right) \delta \theta_{2} \\
a & =R_{\mathrm{e}}\left(2 \cos \theta_{1}-\sin \left(\theta_{1}+\theta_{2}\right)\right) \\
\delta a & =-R_{\mathrm{e}} \cos \left(\theta_{1}+\theta_{2}\right) \delta \theta_{2} .
\end{aligned}
$$

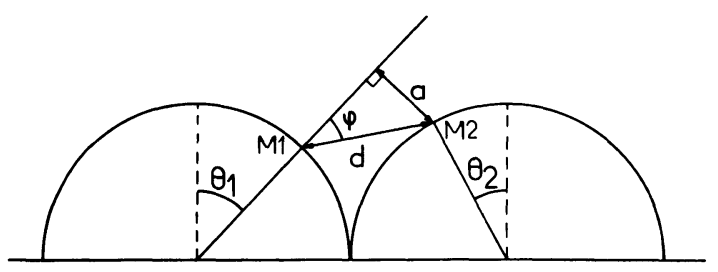

Fig. 3. - Facteur de forme entre deux bandes élémentaires de longueur infinie.

[Configuration factor between two elementary strips of infinite length.]

On peut définir un éclairement adimensionnel par :

$$
E_{\mathrm{v}_{1}}^{*}\left(\theta_{1}\right)=\frac{E_{\mathrm{v}_{1}}\left(\theta_{1}\right)}{\phi_{0}}
$$

l'équation (1) devient alors :

$$
E_{\mathrm{v}_{1}}^{*}\left(\theta_{1}\right)=\cos \theta_{1}+\left(1-\alpha_{\mathrm{v}}\right) \int_{\theta_{2} \text { inf }}^{\theta_{2} \text { up }} E_{\mathrm{v}_{2}}^{*}\left(\theta_{2}\right) \delta F_{\mathrm{d} S_{1} \rightarrow \mathrm{d} S_{2}} .
$$

Or, par raison de symétrie, on a

$$
E_{\mathrm{v}_{1}}^{*}(\theta)=E_{\mathrm{v}_{2}}^{*}(\theta)=E_{\mathrm{v}}^{*}(\theta) .
$$

Il n'y a donc qu'une seule fonction à rechercher en fonction de $\theta$, ce qui est fait numériquement grâce à l'équation implicite :

$$
E_{\mathrm{v}}^{*}\left(\theta_{1}\right)=\cos \theta_{1}+\left(1-\alpha_{\mathrm{v}}\right) \int_{\theta_{2 \mathrm{inf}}}^{\theta_{2} \text { up }} E_{\mathrm{v}}^{*}\left(\theta_{2}\right) \delta F_{\mathrm{d} S_{1} \rightarrow \mathrm{d} S_{2}} .
$$

Il suffit d'initialiser $E_{\mathrm{v}}^{*}(\theta)$ par la fonction $\cos \theta$ et de faire un calcul itératif pour avoir la précision souhaitée. Ce qui conduit à la répartition du flux visible absorbé $\phi_{\mathrm{v}}(\theta)$.

2.2 CAlCUl Des Températures À L'INTÉRIEUR DU TUBE. - Si l'on suppose connue la répartition du flux global (visible et infrarouge) absorbé, on peut calculer, moyennant les hypothèses faites précédemment, la température à n'importe quel point de la section droite du tube en résolvant l'équation de la chaleur : 


$$
\frac{\partial^{2} T}{\partial r^{2}}+\frac{1}{r} \frac{\partial T}{\partial r}+\frac{1}{r^{2}} \frac{\partial^{2} T}{\partial \theta^{2}}=0 \quad R_{\mathrm{i}} \leqslant r \leqslant R_{\mathrm{e}} \text { et } \quad 0 \leqslant \theta \leqslant 2 \pi
$$

avec les conditions aux limites associées :

$$
\begin{array}{ll}
k \frac{\partial T}{\partial r}=h\left(T-T_{\mathrm{v}}\right) & r=R_{\mathrm{i}} \text { et } 0 \leqslant \theta \leqslant 2 \pi \\
k \frac{\partial T}{\partial r}=\phi(\theta)=\phi_{\mathrm{v}}(\theta)+\phi_{\mathrm{ir}}(\theta) & r=R_{\mathrm{c}} \text { et } \quad 0 \leqslant \theta \leqslant 2 \pi
\end{array}
$$

où $k$ est la conductivité thermique du métal et $\phi(\theta)$ le flux global absorbé qu’on peut décomposer en série de Fourier :

$$
\phi(\theta)=\phi_{0}\left(\frac{a_{0}}{2}+\sum_{n=1}^{+\infty} a_{n} \cos n \theta+b_{n} \sin n \theta\right)
$$

On peut passer aux variables adimensionnées suivantes :

$$
\psi=\frac{k\left(T-T_{\mathrm{v}}\right)}{R_{\mathrm{i}} \phi_{0}} ; \quad \mu=\frac{r}{R_{\mathrm{i}}} ; \quad B=\frac{h R_{\mathrm{i}}}{k} ; \quad \mu_{n}=\frac{R_{\mathrm{e}}}{R_{\mathrm{i}}} .
$$

Le système précédent devient alors :

$$
\begin{aligned}
& \frac{\partial^{2} \psi}{\partial \mu^{2}}+\frac{1}{\mu} \frac{\partial \psi}{\partial \mu}+\frac{1}{\mu^{2}} \frac{\partial^{2} \psi}{\partial \theta^{2}}=0 \quad \text { pour } \quad 1 \leqslant \mu \leqslant \mu_{n} \text { et } 0 \leqslant \theta \leqslant 2 \pi \\
& \frac{\partial \psi}{\partial \mu}=\frac{a_{0}}{2}+\sum_{n=1}^{+\infty}\left(a_{n} \cos n \theta+b_{n} \sin n \theta\right) \quad \mu=\mu_{n} \quad \text { et } \quad 0 \leqslant \theta \leqslant 2 \pi \\
& \frac{\partial \psi}{\partial \mu}=B \psi \quad \mu=1 \quad \text { et } \quad 0 \leqslant \theta \leqslant 2 \pi .
\end{aligned}
$$

La solution de ce problème est de la forme :

$$
\psi(\mu, \theta)=\frac{a_{0}}{2} \mu_{n} \log \mu+\frac{a_{0}}{2} \frac{\mu_{n}}{B}+\sum_{p=1}^{+\infty}\left(A_{p} \mu^{p}+B_{p} \mu^{-p}\right) \cos p \theta+\left(A_{p}^{\prime} \mu^{p}+B_{p}^{\prime} \mu^{-p}\right) \sin p \theta
$$

avec

$$
A_{p}=\frac{a_{p}(p+B) \mu_{n}^{p+1}}{p\left[(p+B) \mu_{n}^{2 p}-p+B\right]} ; \quad B_{p}=\frac{a_{p}(p-B) \mu_{n}^{p+1}}{p\left[(p+B) \mu_{n}^{2 p}-p+B\right]}
$$

$A_{p}^{\prime}$ et $B_{p}^{\prime}$ sont donnés par les mêmes relations en remplaçant $a_{p}$ par $b_{p}$.

Dans le cas qui nous concerne, $\phi(\theta)$ étant une fonction paire, on a :

$$
\forall i \in \mathbb{N}^{*}, \quad b_{i}=0
$$

et par conséquent $A_{i}^{\prime}=B_{i}^{\prime}=0$.

Il ne reste donc que les termes en cosinus dans l'expression de $\psi(\mu, \theta)$.

Mais la connaissance de $\phi(\theta)$ nécessite elle-même la connaissance des températures pour pouvoir accéder au flux infrarouge $\phi_{\mathrm{ir}}(\theta)$ absorbé localement. Nous allons voir comment on peut avoir $\phi_{\mathrm{ir}}(\theta)$ en fonction de $T\left(r_{\mathrm{e}}, \theta\right)$, qu'on notera plus simplement $T_{\mathrm{e}}(\theta)$ par la suite.

2.3 Radiosités infrarouges. - Avec les mêmes notations que celles de la figure 2 , la radiosité infrarouge, ou flux surfacique partant de la bande passant par $\mathbf{M}_{1}$, se calcule par la relation :

$$
\begin{aligned}
J_{1}\left(\theta_{1}\right)=\varepsilon_{\mathrm{ir}} & \sigma\left(T_{\mathrm{e}}\left(\theta_{1}\right)\right)^{4}+ \\
& +\left(1-\alpha_{\mathrm{ir}}\right) \int_{\theta_{2 \mathrm{inf}}}^{\theta_{2} \text { up }} J_{2}\left(\theta_{2}\right) \delta F_{\mathrm{d} S_{1} \rightarrow \mathrm{d} S_{2}} .
\end{aligned}
$$

En effet, $J_{1}\left(\theta_{1}\right)$ est la somme du flux directement émis du fait de la température $T_{\mathrm{e}}\left(\theta_{1}\right)$ (le domaine de température de paroi considéré autorise à assimiler l'émission propre dans la bande infrarouge à l'émission totale) et du flux I.R. venant des surfaces avoisinantes et réfléchi en $\mathbf{M}_{1}$. 
A cause toujours de la symétrie du problème, on a :

$$
J_{1}(\theta)=J_{2}(\theta)=J(\theta)
$$

ce qui nous ramène à l'équation implicite :

$$
\begin{aligned}
& J\left(\theta_{1}\right)=\varepsilon_{\mathrm{ir}} \sigma\left(T_{\mathrm{e}}\left(\theta_{1}\right)\right)^{4}+ \\
& +\left(1-\alpha_{\mathrm{ir}}\right) \int_{\theta_{2 \mathrm{inf}}}^{\theta_{2 \mathrm{up}}} J\left(\theta_{2}\right) \delta F_{\mathrm{d} S_{1} \rightarrow \mathrm{d} S_{2}}
\end{aligned}
$$

où $\theta_{2 \text { inf }}, \theta_{2 \text { sup }}$ et $\delta F_{\mathrm{d} S_{1} \rightarrow \mathrm{d} S_{2}}$ sont donnés respectivement par les relations (2), (3) et (4).

L'équation (8) est tout à fait similaire à (5) et sera résolue numériquement de la même façon, il suffit de remplacer $\alpha_{\mathrm{v}}$ par $\varepsilon_{\mathrm{ir}}$ et d'initialiser $J\left(\theta_{1}\right)$ par $\varepsilon_{\text {ir }} \sigma\left(T_{\mathrm{e}}\left(\theta_{1}\right)\right)^{4}$.

Une fois connue la radiosité infrarouge, on peut calculer le flux surfacique gagné en $M_{1}$ :

$$
\begin{aligned}
\phi_{\mathrm{ir}}\left(\theta_{1}\right) & =\alpha_{\mathrm{ir}} \int_{\theta_{2 \mathrm{inf}}}^{\theta_{2} \text { up }} J\left(\theta_{2}\right) \delta F_{\mathrm{d} S_{1} \rightarrow \mathrm{d} S_{2}}-\varepsilon_{\mathrm{ir}} \sigma\left(T_{\mathrm{e}}\left(\theta_{1}\right)\right)^{4} \\
& =\frac{\alpha_{\mathrm{ir}}}{1-\alpha_{\mathrm{ir}}}\left(J\left(\theta_{1}\right)-\varepsilon_{\mathrm{ir}} \sigma\left(T_{\mathrm{e}}\left(\theta_{1}\right)\right)^{4}\right)-\varepsilon_{\mathrm{ir}} \sigma\left(T_{\mathrm{e}}\left(\theta_{1}\right)\right)^{4} \quad \text { d'après (8) }
\end{aligned}
$$

Or, comme on a supposé d'une part que les substances étaient grises dans la bande I.R. et d'autre part que la réflexion et l'émission étaient isotropes, on a :

d'où :

$$
\alpha_{\mathrm{ir}}=\varepsilon_{\mathrm{ir}}
$$

$$
\phi_{\mathrm{ir}}\left(\theta_{1}\right)=\frac{\varepsilon_{\mathrm{ir}}}{1-\varepsilon_{\mathrm{ir}}}\left(J\left(\theta_{1}\right)-\sigma\left(T_{\mathrm{e}}\left(\theta_{1}\right)\right)^{4}\right) .
$$

Et le flux surfacique global gagné en $M_{1}$ est donné par :

$$
\phi\left(\theta_{1}\right)=\phi_{\mathrm{v}}\left(\theta_{1}\right)+\phi_{\mathrm{ir}}\left(\theta_{1}\right)=\alpha_{\mathrm{v}} \phi_{0} E_{\mathrm{v}}^{*}\left(\theta_{1}\right)+\frac{\varepsilon_{\mathrm{ir}}}{1-\varepsilon_{\mathrm{ir}}}\left(J\left(\theta_{1}\right)-\sigma\left(T_{\mathrm{e}}\left(\theta_{1}\right)\right)^{4}\right) .
$$

Par itération entre les relations (6), (8) et (10), nous pouvons à présent déterminer les fonctions $T_{\mathrm{e}}(\theta), J(\theta)$, $\phi(\theta), T(r, \theta), \ldots$ avec la précision que l'on désire.

2.4 Flux infrarouge Partant De La Parol. Avant de calculer le flux surfacique et hémisphérique infrarouge quittant la surface tubulaire, il faut exprimer la puissance infrarouge partant d'une bande élémentaire dans un angle solide délimité par un dièdre plan qu'on appellera «flux pseudo-directionnel ».

Pour cela, soient (voir Fig. 4) :

- $\mathrm{d} S$ une surface élémentaire de la bande de longueur infinie notée $S$.

- $\mathbf{n}$ le vecteur unitaire normal à $\mathrm{d} S$. de $S$.

- $P_{N}$ le plan contenant $n$ et la droite génératrice

- $\mathrm{P}(\delta)$ et $\mathrm{P}(\delta+\mathrm{d} \delta)$ les deux demi-plans formant avec $\mathbf{P}_{\mathbf{N}}$ deux dièdres dont l'arête commune est la génératrice de $S$ et les angles plans $\delta$ et $\delta+\mathrm{d} \delta$.

Le flux partant de la surface élémentaire $\mathrm{d} S$ vers la surface élémentaire $\mathrm{d} S^{\prime}$ est donné par :

$$
\mathrm{d}^{4} \phi=L \cos (\mathbf{n}, \mathbf{O M}) \mathrm{d} S . \mathrm{d} \Omega
$$

$\mathrm{d} \Omega$ étant l'angle solide sous lequel $\mathrm{d} S^{\prime}$ est vue de $\mathrm{d} S$ et $L$ la luminance du flux partant de $\mathrm{d} S$

$$
\mathrm{d} \Omega=\mathrm{d} S^{\prime} \frac{\mathbf{O M} \cdot \mathbf{n}^{\prime}}{\|\mathbf{O M}\|^{3}}
$$

où $\mathbf{n}^{\prime}$ est le vecteur unitaire normal à $\mathrm{d} S^{\prime}$.

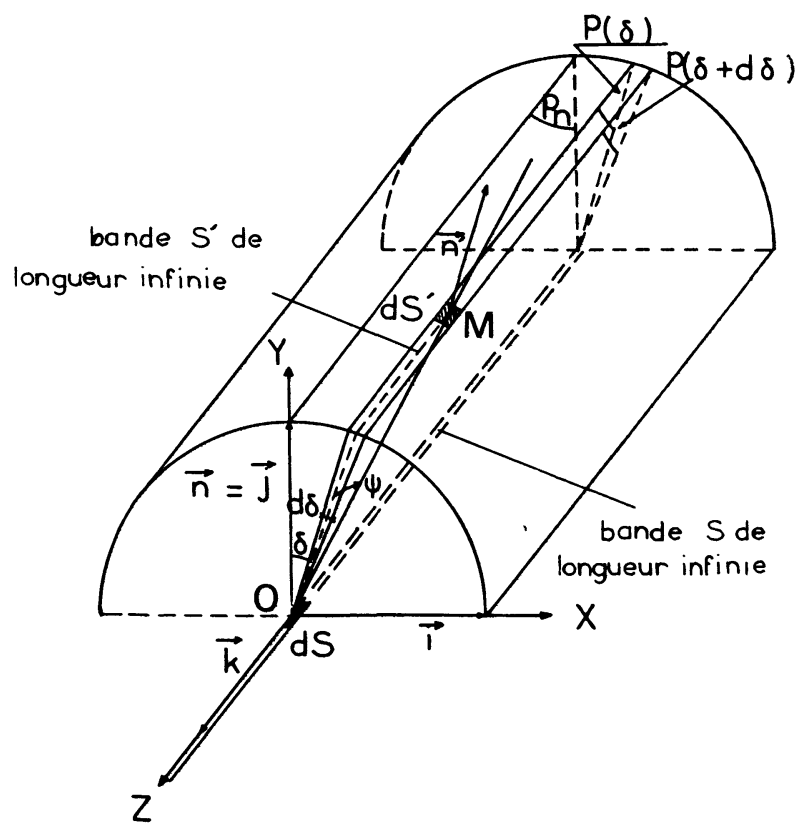

Fig. 4. - Emission d'une surface élémentaire $\mathrm{d} S$ dans un dièdre plan $[\mathrm{P}(\delta), \mathrm{P}(\delta+\mathrm{d} \delta)]$.

[Emission of an elementary area $\mathrm{d} S$ in a plane dihedron $[\mathrm{P}(\delta), \mathrm{P}(\delta+\mathrm{d} \delta)]$. 
Soit

$$
\mathrm{d}^{4} \phi=L \frac{\mathbf{O M . n}}{\|\mathbf{O M}\|} \cdot \frac{\mathbf{O M} \cdot \mathbf{n}^{\prime}}{\|\mathbf{O M}\|^{3}} \mathrm{~d} S \cdot \mathrm{d} S^{\prime}
$$

avec :

$$
\begin{aligned}
\mathbf{O M} & =\sin \delta \mathbf{i}+\cos \delta \mathbf{j}+z \mathbf{k} \\
\mathbf{n} & =\mathbf{j} \\
\mathbf{n}^{\prime} & =\sin \delta \mathbf{i}+\cos \delta \mathbf{j}
\end{aligned}
$$

d'où

$$
\mathrm{d}^{4} \phi=L \cos \delta \mathrm{d} \delta \frac{\mathrm{d} z}{\left(1+z^{2}\right)^{2}} \mathrm{~d} S .
$$

Si $\psi$ est l'angle que fait $\mathbf{O M}$ avec sa projection sur le $\operatorname{plan}(0, \mathbf{i}, \mathbf{j})$ on a $z=\operatorname{tg} \psi$ et

$$
\mathrm{d}^{4} \phi=L \cdot \cos \delta \cdot \mathrm{d} \delta \cdot \cos ^{2} \psi \cdot \mathrm{d} \psi \cdot \mathrm{d} S .
$$

Le flux partant de $\mathrm{d} S$ dans tout le dièdre $[\mathrm{P}(\delta), \mathrm{P}(\delta+\mathrm{d} \delta)]$ s'exprime par :

$$
\mathrm{d}^{3} \phi=\cos \delta . \mathrm{d} \delta . \mathrm{d} S \int_{-\pi / 2}^{\pi / 2} L \cos ^{2} \psi \mathrm{d} \psi .
$$

Or, dans notre cas, l'isotropie de l'émission et de la réflexion fait que la luminance du flux partant de $\mathrm{d} S$ ne dépend pas de $\psi$, d'où après intégration :

$$
\mathrm{d}^{3} \phi=\frac{\pi}{2} L \cos \delta \mathrm{d} \delta \mathrm{d} S .
$$

Si l'on applique maintenant ce résultat à la paroi tubulaire, le flux pseudo-directionnel infrarouge partant d'une surface de $2 \times R_{\mathrm{e}} \times 1 \mathrm{~m}^{2}$ (pour une unité de longueur des tubes) entre les directions $\gamma$ et $\gamma+\mathrm{d} \gamma$ s'écrit :

$$
\begin{aligned}
\mathrm{d} \phi=\left(\int_{\theta_{1 \mathrm{i}}}^{\theta_{1 s}} \frac{\pi}{2} L\left(\theta_{1}\right) \cos \delta_{1} R_{\mathrm{e}} \mathrm{d} \theta_{1}+\right. \\
\left.\quad+\int_{\theta_{2 \mathrm{i}}}^{\theta_{2 \varsigma}} \frac{\pi}{2} L\left(\theta_{2}\right) \cos \delta_{2} R_{\mathrm{e}} \mathrm{d} \theta_{2}\right) \mathrm{d} \gamma
\end{aligned}
$$

où $L\left(\theta_{1}\right)$ est la luminance du flux infrarouge partant de la bande passant par $M_{1}$ :

$$
L\left(\theta_{1}\right)=\frac{J\left(\theta_{1}\right)}{\pi} ; \quad L\left(\theta_{2}\right)=\frac{J\left(\theta_{2}\right)}{\pi} ;
$$

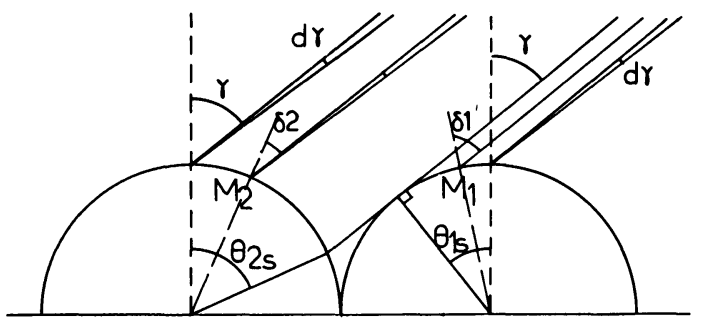

Fig. 5. - Emission pseudo-directionnelle du faisceau de tubes.

[Pseudodirectional emission of the bundle of tubes.] et $\delta_{1}$ et $\delta_{2}$ sont les angles entre la direction de l'émission et les normales locales en $\mathbf{M}_{1}$ et $\mathbf{M}_{2}$ (Fig. 5).

$$
\begin{aligned}
& \delta_{1}=\theta_{1}+\gamma \\
& \delta_{2}-\theta_{2}+\gamma .
\end{aligned}
$$

Quant aux angles limites $\theta_{1 \mathrm{i}}, \theta_{1 \mathrm{~s}}, \theta_{2 \mathrm{i}}$ et $\theta_{2 \mathrm{~s}}$ ils sont donnés par :

$$
\begin{aligned}
& \theta_{1 \mathrm{i}}=0 \\
& \theta_{1 \mathrm{~s}}=\frac{\pi}{2}-\gamma \\
& \theta_{2 \mathrm{i}}=0 \\
& \theta_{2 \mathrm{~s}}=\gamma+\operatorname{Arcsin}(2 \cos \gamma-1) .
\end{aligned}
$$

Le flux surfacique hémisphérique infrarouge partant de la surface tubulaire peut enfin s'écrire :

$$
\phi_{\mathrm{t}}=\frac{1}{2 R_{\mathrm{e}}} \int_{-\pi / 2}^{\pi / 2} \frac{\mathrm{d} \phi}{\mathrm{d} \gamma} \mathrm{d} \gamma
$$

soit

$$
\begin{aligned}
\phi_{\mathrm{t}}=\frac{1}{2 R_{\mathrm{e}}} \int_{-\pi / 2}^{\pi / 2} & \left(\int_{\theta_{1 \mathrm{i}}}^{\theta_{1} \iota} \frac{J\left(\theta_{1}\right)}{2} \cos \delta_{1} R_{\mathrm{e}} \mathrm{d} \theta_{1}+\right. \\
& \left.+\int_{\theta_{2 \mathrm{i}}}^{\theta_{2 \iota}} \frac{J\left(\theta_{2}\right)}{2} \cos \delta_{2} R_{\mathrm{e}} \mathrm{d} \theta_{2}\right) \mathrm{d} \gamma
\end{aligned}
$$

que l'on peut calculer grâce à la radiosité $J(\theta)$ trouvée précédemment.

3. Résultats. - Ce calcul itératif a été d'abord effectué avec les valeurs numériques correspondant au paquet de tubes recevant le maximum de flux solaire dans la chaudière de la centrale électrosolaire Themis :

$$
\begin{aligned}
\phi_{0} & =733 \mathrm{~kW} / \mathrm{m}^{2}, \\
T_{\mathrm{v}} & =540 \mathrm{~K}, \\
h & =7015 \mathrm{~W} / \mathrm{m}^{2}{ }^{\circ} \mathrm{C}, \\
k & =20 \mathrm{~W} / \mathrm{m}^{\circ} \mathrm{C}, \\
R_{\mathrm{e}} & =9 \mathrm{~mm}, \\
R_{\mathrm{i}} & =7,5 \mathrm{~mm} .
\end{aligned}
$$

Les coefficients radiatifs sont ceux du revêtement Pyromark, utilisé sur la même chaudière et qui présente l'avantage d'être très absorbant mais aussi l'inconvénient d'avoir une émissivité très forte :

$$
\alpha_{v}=\varepsilon_{i r}=0,95 \text { (Réf. [4]). }
$$

La figure 6 représente la répartition des températures dans le tube d'abord sans tenir compte de l'émission et de la redistribution des flux : (a) $\left(\phi=\alpha_{v} \phi_{0} \cos \theta\right)$ et puis en tenant compte de ces deux phénomènes : $(b)$.

Il apparaît sur cette figure une différence de tempé- 


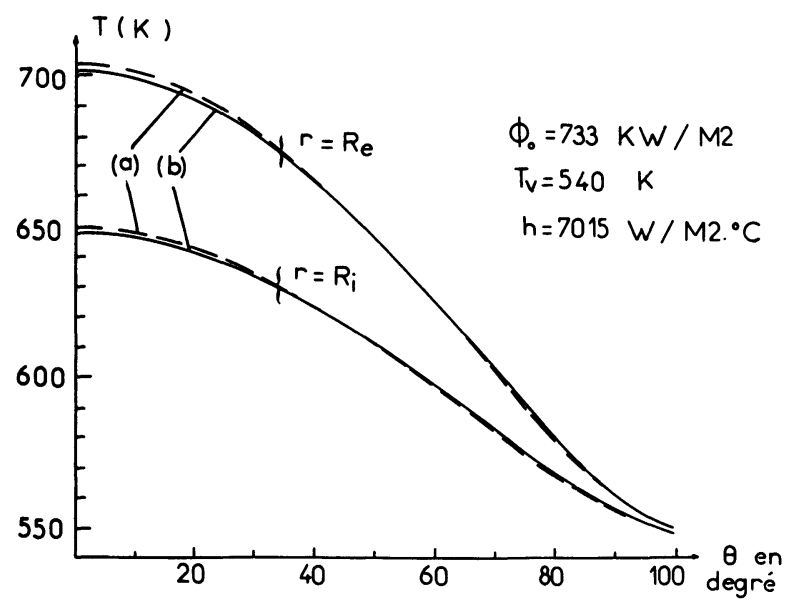

Fig. 6. - Températures dans le tube. (a) : sans tenir compte de l'émission et de la redistribution $(--) ;(b)$ : en tenant compte (-).

[Temperature profile in the tube : $(a):$ without taking into account the emission and redistribution effects $(--)$; (b) : taking into account these effects (-).]

ratures de l'ordre de $150^{\circ} \mathrm{C}$ entre le sommet et la base du tube. Ce fort gradient est dû à la valeur de $\phi_{0}$ qui est très élevée. Par contre, l'écart entre les deux courbes $(a)$ et $(b)$ reste assez faible, ce qui résulte de deux causes :

- Le flux incident est très grand devant les flux émis ou redistribués.

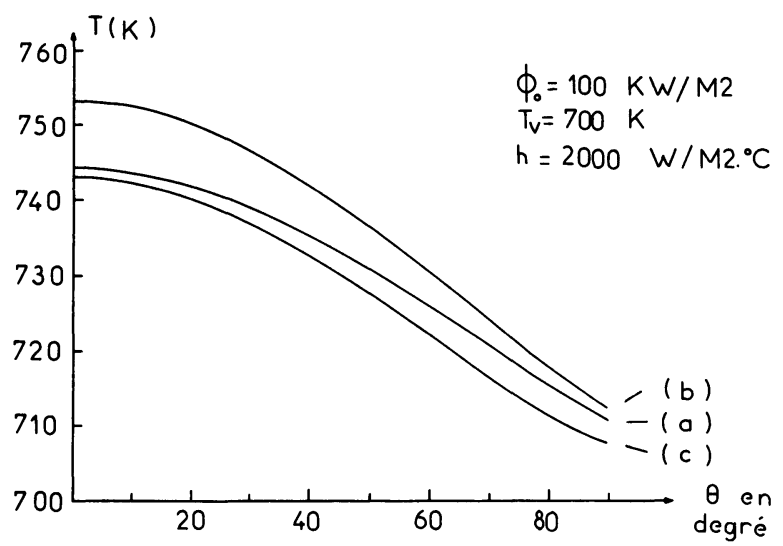

Fig. 6 bis. - Températures à la paroi externe du tube; $(a)$ : en tenant compte de tout $(\phi(\theta)$ exact $) ;(b)$ : en ne tenant compte ni de l'émission ni de la redistribution

$$
\left(\phi(\theta)=\alpha_{v} \phi_{0} \cos \theta\right) ;
$$

(c) : en tenant compte de l'émission uniquement

$$
\left(\phi(\theta)=\alpha_{\mathrm{v}} \phi_{0} \cos \theta-\varepsilon_{\mathrm{ir}} \sigma\left(T_{\mathrm{e}}(\theta)\right)^{4}\right) .
$$

[Temperature profile in the external tube wall; $(a):$ taking into account the emission and redistribution effects $(\phi(\theta)$ exact); $(b)$ : without taking into account the emission and redistribution effects $\left(\phi(\theta)=\alpha_{v} \phi_{0} \cos \theta\right) ;(c)$ : taking into account only the emission effect

$$
\left.\left(\phi(\theta)=\alpha_{\mathrm{v}} \phi_{0} \cos \theta-\varepsilon_{\mathrm{ir}} \sigma\left(T_{\mathrm{e}}(\theta)\right)^{4}\right) .\right]
$$

- Les facteurs d'absorption visible et infrarouge sont très élevés, ce qui diminue notablement l'influence de la redistribution puisque la presque totalité du flux qui arrive en un point y est absorbée.

La figure 6 bis représente les mêmes courbes de température pour une valeur de $\phi_{0}$ plus faible et une température de veine fluide plus élevée. Le gradient est alors plus faible alors que l'émission du tube et la redistribution prennent plus d'importance.

La figure 7 nous montre, pour les valeurs numériques précédentes, la comparaison entre les flux surfaciques infrarouge et visible redistribués et le flux surfacique émis, on y trouve la répartition angulaire des trois grandeurs suivantes :

- Le flux surfacique infrarouge émis en $M_{1}$;

- Le flux surfacique infrarouge venant du tube 2 et absorbé en $\mathbf{M}_{1}$;

- Le flux surfacique visible venant du tube 2 et absorbé en $\mathbf{M}_{1}$.

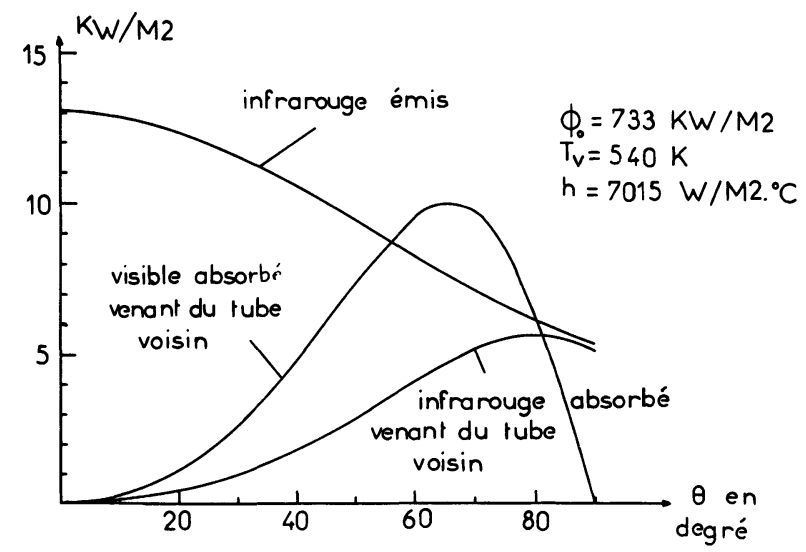

Fig. 7. - Importance de la redistribution vis-à-vis de l'émission.

[Redistribution compared to emission.]

On peut noter que malgré les fortes valeurs de $\alpha_{v}$ et $\alpha_{i r}$, l'émission est largement compensée par la redistribution à partir d'un angle $\theta$ égal à $45^{\circ}$ environ.

Quant au flux hémisphérique infrarouge partant d'une unité de surface de faisceau de tubes, on peut le caractériser par une température qui serait celle d'un échantillon plan ayant les mêmes caractéristiques radiatives que la surface externe des tubes, pour émettre le même flux, soit :

$$
\phi_{\mathrm{t}}=\varepsilon_{\mathrm{ir}} \sigma T_{\mathrm{rad}}^{4} .
$$

Avec les données numériques précédentes, on obtient : $T_{\mathrm{rad}}=681 \mathrm{~K}$.

La figure 8 compare les deux fonctions suivantes :

- Le flux pseudo-directionnel surfacique : $\frac{1}{2 R_{\mathrm{e}}} \frac{\mathrm{d} \phi}{\mathrm{d} \gamma}$.

- Le flux pseudo-directionnel surfacique émis par une surface plane de même émissivité $\varepsilon_{\mathrm{ir}}$ et à la température équivalente radiative $T_{\text {rad }}$. 
Flux pseudo-directionnel

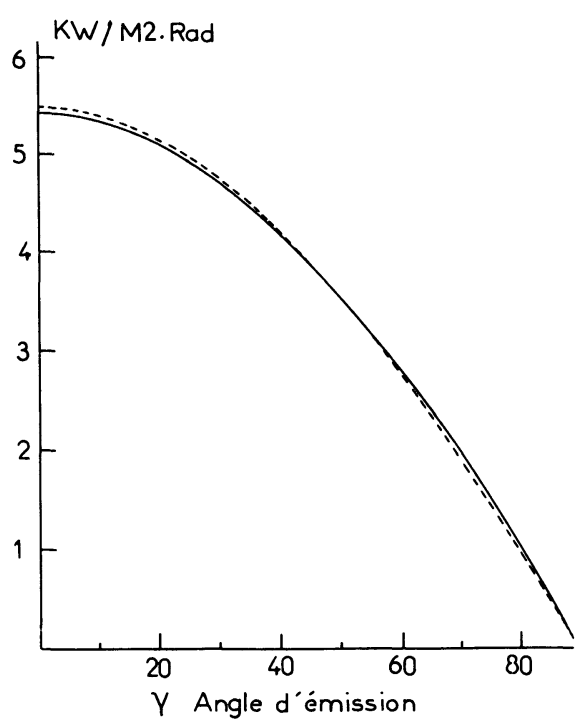

Fig. 8. - Flux pseudo-directionnel surfacique émis par : (a) : le faisceau réel de tubes $(-) ;(b)$ : une surface plane à $T_{\text {rad }}$ et d'émissivité isotrope $\varepsilon_{\text {ir }}(---)$.

[Density of pseudo-directional flux emitted by : $(a):$ the real bundle of tubes $(-) ;(b):$ a plane surface at $T_{\mathrm{rad}}$ and with an isotropic emissivity (- - - ).]

Il est intéressant de noter sur cette figure que, même avec un très fort gradient de température dans le tube, l'émission globale reste isotrope avec une très bonne approximation, malgré toutefois une légère augmentation par rapport à l'échantillon plan pour les angles rasants et une légère diminution pour les angles normaux. Ces petites différences sont dues au maximum de température situé au sommet du tube qui est justement vu pour les angles rasants.

Il nous a semblé intéressant à la suite de l'étude de ce cas numérique, de faire une étude paramétrique de $T_{\text {rad }}$. Mais étant donné le grand nombre de paramètres qui rentrent en jeu, on s'est limité aux trois suivants :

$-\phi_{\text {abs }}$ : flux global moyen absorbé par le paquet de tubes. On le fera varier de 0 à $750 \mathrm{~kW} / \mathrm{m}^{2}$.

$-T_{\mathrm{v}}$ : température de veine fluide qu'on fera varier entre 550 et $700 \mathrm{~K}$.

$-h$ : coefficient d'échange tube fluide compris entre 2000 et $8000 \mathrm{~W} / \mathrm{m}^{2}{ }^{\circ} \mathrm{C}$.

A $h$ constant, il apparaît des corrélations linéaires du type :

$$
T_{\mathrm{rad}}-T_{\mathrm{v}}=\alpha \phi_{\mathrm{abs}}+\beta
$$

où $\alpha$ a la dimension d'une résistance thermique et $\beta$ celle d'une température.

La figure 9 représente les variations de $\alpha$ en fonction de $h$, qu'on peut interpoler avec une très bonne approximation par la fonction

$$
\alpha=\left(\frac{1}{h}+\frac{e}{k}\right) \cdot A+B_{\mathrm{r}} \text { avec } \begin{aligned}
& A=0,764 \\
& B_{\mathrm{r}}=3 \times 10^{-5}
\end{aligned} .
$$

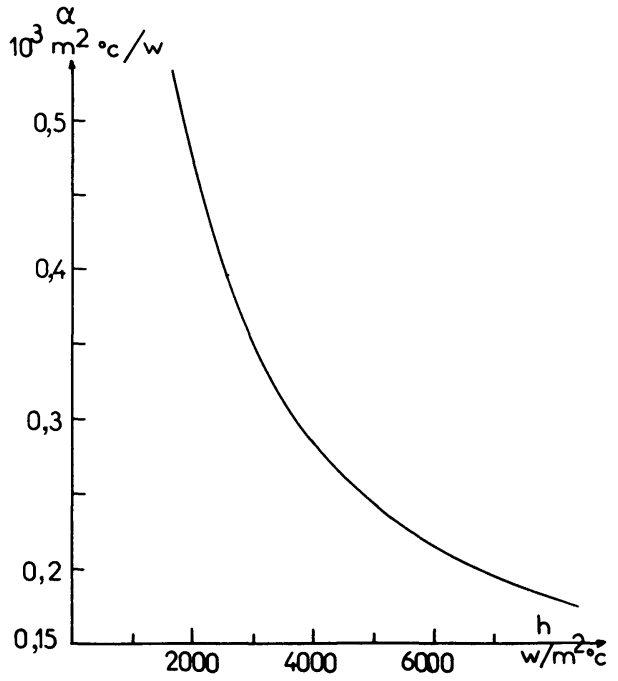

Fig. 9. - Variation de $\alpha$ (résistance thermique) en fonction de $h$.

[ $\alpha$ (thermal resistance) as a fonction of $h$.]

Quant à $\beta$, elle ne varie pratiquement pas en fonction de $h$ et reste égale à $4^{\circ} \mathrm{C}$.

4. Conclusions. - On peut caractériser la surface externe de la paroi tubulaire par deux températures, l'une $T_{\mathrm{cc}}$ servant aux échanges conductifs et convectifs et qui est donnée par :

$$
T_{\mathrm{cc}}-T_{\mathrm{v}}=A\left(\frac{1}{h}+\frac{e}{k}\right) \phi_{\mathrm{abs}} .
$$

L'autre servant aux échanges radiatifs :

$$
T_{\mathrm{rad}}-T_{\mathrm{cc}}=B_{\mathrm{r}} \phi_{\mathrm{abs}}+\beta .
$$

Il apparaît ainsi une notion de résistance thermique radiative, si on peut l'appeler ainsi, qui caractérise la macrorugosité de la surface et qui dépend bien sûr des caractéristiques radiatives $\alpha_{\mathrm{v}}$ et $\varepsilon_{\mathrm{ir}}$. La différence entre ces deux températures est d'autant plus forte que la valeur du flux incident est élevée. Et l'on peut commettre des erreurs non négligeables en se servant de $T_{\text {cc }}$ dans des calculs d'échanges radiatifs.

Cette étude a permis de montrer d'un autre côté, que si l'on suppose l'isotropie locale de l'émission et de la réflexion, cette propriété se conserve à une échelle plus grande que celle des macrorugosités, du moins pour la géométrie particulière qui a été étudiée.

Enfin, l'utilisation du concept de température équivalente radiative, qui remplace celui d'émissivité apparente pour une paroi macrorugueuse isotherme, est rendue aisée grâce à la paramétrisation précédente ne faisant intervenir que des expressions simples. 


\section{Bibliographie}

[1] Druelle, P., "Influence de macrorugosités sur les caractéristiques radiatives de certaines surfaces ", Thèse de Docteur Ingénieur soutenue à l'Ecole Centrale de Paris (octobre 1978).

[2] Pietri, P., « Etude de quelques problèmes de contraintes thermiques dans les tubes de chaudières de centrales électrosolaires ", Thèse de Docteur Ingénieur soutenue à Poitiers (septembre 1980).

[3] Reynolds, W. C., "Turbulent heat transfer in a circular tube with variable circumferential heat flux ». Int. J. Heat Mass Trans. 6 (1963) 445-454.
[4] Stekelorom, P., «Etude de la peinture Pyromark 2500 », Rapport E.D.F., Ecole Centrale de Paris (novembre 1981).

[5] Tran N'GuYen, H., "Calcul des propriétés radiatives de surfaces macro-rugueuses par la méthode de Monte Carlo ", Thèse de Docteur Ingénieur soutenue à l'Ecole Centrale de Paris (juin 1981). 